\title{
Non Stationary Ionic Current through Polymer Charged Membrane
}

\author{
Sunghyun Jeong, ${ }^{*}$ Wonchul Lee, and Wongkang Yang ${ }^{\star}$ \\ College of Oriental Medicine, Dongguk Vhiversity. Gvongiu 780-714. Korea \\ "Department of Chemistry, College of Ratural Science, Dongguk University. Gvongiu 780-714. Korea \\ Received Mav 20,2003
}

\begin{abstract}
The relaxation phenomena of ionic currents through the charged membrane under the constant applied potentials has been studied. The fornulation was obtained for the non stationary current by assuming that the ion mobility is independent of concentration and the potential gradient is a constant within membrane. and it was applied to the experimental results with the sulfonated polystyrene collodion base membrane. It has been shown that the initial ion distributions in the membrane play a predominant role in the relavation phenomena.
\end{abstract}

Key Words : Relaxation phenomena. Ionic current, Potential gradient. Collodion membrane, Ion distribution

\section{Introduction}

The time course of ionic currents through the excitable membranes have been well described by reversible changes in sodium and potassium conductances. ${ }^{1}$ More information concerning the change in the ionic conductance were obtained by the techniques of intercellular perfusion and the behavior of various ions other than sodiun ion and potassium ion were discussed? ? $^{2}$

Since the alteration in the ion conductances may attributed to the change of ion concentration in the composite charged membrane. the relaxation of ionic currents during voltage clamp would be interpreted by the time dependent redistribution of ions within membrane provided that the passive ion movements govern the processes. ${ }^{3}$ Therefore. it is of important to find out the essential feature of non-steady ion current under a constant voltage with the artificial mentbrane electrolytes system Through the exact description for the non-stationary state of the membrane electrolyte system were given by Kirkwood. ${ }^{A}$ it is generally impossible to estimate the ionic current as the function of applied voltage and ionic concentration without the knowledge of the detailed structure of the system. Hence. it is necessary to make simple assumption in order to express the non-steady current in terms of the simple measurable quantities such as the applied voltage and the salt concentration of exterior sides of membrane.

In this study: the assumption of a constant electric field was applied to the non-stationary problem. Additional assumptions are that the ionic mobilities are independent of ionic concentration and there is no cross term in diffusion coefficient. On the basis of these assumption. an expression for the non-steady ionic current was derived and was applied to the experimental data obtained with artificial systems.

\section{Experimental Section}

The membrane was dried type sulfonated polystyrene collodion base membrane prepared from $3.5 \%$ collodion solution containing $0.85 \% / \mathrm{L}$ sulfonated polystyrene. ${ }^{5}$ The thickness of membrane was $0.03 \mathrm{~mm}$. The measurements of membrane conductance were made by d.c. method using the cell devised by Laksminarayanaiah. ${ }^{5}$ After the membrane was equilibrated with exterior electrolyte solutions. a potential was applied across membrane with the current carrying silver-silver chloride electrode. The applied potential was kept constant by using a variable resistor and silver-silver chloride probes operated to the detect the membrane potential. The change in the currents with time were recorded as the potential depression between a standard resistance.

The calomel electrodes were used to determined the resting potential. The experiments were carried at $25^{\circ} \mathrm{C}$ without a perfusion of electrolyte solutions.

\section{Results and Discussion}

The typical time course of non-steady currents with a various applied voltages for the system which consists of the membrane fixed between the $0.01 \mathrm{~N}-\mathrm{KCl}$ solution and the $0.01 \mathrm{~N}-\mathrm{CaCl}_{2}$ solution are illustrated in Figure 1. This system has the resting potential of $35.6 \mathrm{mV}$. The currents decreased with time when the initial applied potential was displaced to the anodal direction. and the relaxation times as well as the amount of variation in the current increased as the applied potentials become larger. On the other hand. the current increased during the course of the record when the potential deviations from the resting value were negative. In

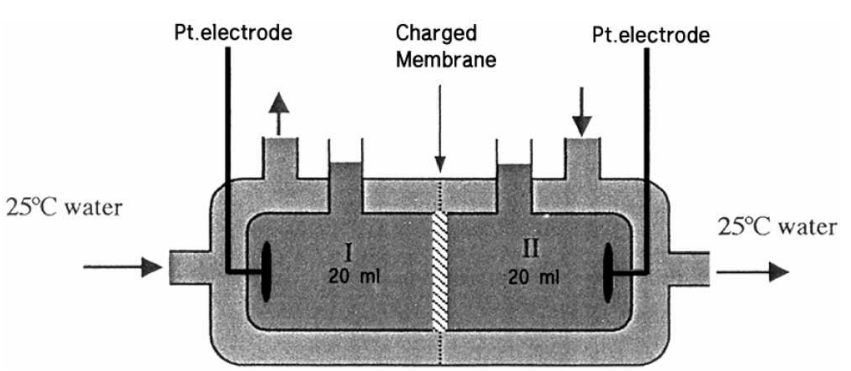

Figure 1. A schematic apparatus for ionic conductivity measurement. 
Table 1. The membrane potential, membrane conductance, and membrane penneability at resting state through composite charged membrane and system no. $1: 0.01 \mathrm{~N}-\mathrm{KCl} / 0.001 \mathrm{~N}-\mathrm{KCl}, 2: 0.01 \mathrm{~N}-\mathrm{KCl} / 0.001 \mathrm{~N}-\mathrm{CaCl}_{2}, 3: 0.01 \mathrm{~N}-\mathrm{KCl} / 0.01 \mathrm{~N}-\mathrm{CaCl}, 4: 0.01 \mathrm{~N}-\mathrm{KCl} / 0.1 \mathrm{~N}-$ $\mathrm{CaCl}_{2}$

\begin{tabular}{cccccccc}
\hline $\begin{array}{c}\text { System } \\
110 .\end{array}$ & $\begin{array}{c}\mathrm{V}_{\mathrm{D}} \\
(\mathrm{mV})\end{array}$ & $\begin{array}{c}\mathrm{G} \\
\left(\mathrm{m} / 10 / \mathrm{cm}^{2}\right)\end{array}$ & $\begin{array}{c}\mathrm{G}_{+} \\
\left(\mathrm{m} h \mathrm{~h} / \mathrm{cm}^{2}\right)\end{array}$ & $\begin{array}{c}\mathrm{G}_{-} \\
\left(\mathrm{mho} / \mathrm{cm}^{2}\right)\end{array}$ & $\begin{array}{c}\mathrm{P}_{\mathrm{K}} \times 10^{5} \\
(\mathrm{~cm} / \mathrm{sec})\end{array}$ & $\begin{array}{c}\mathrm{P}_{(\mathrm{a}} \times 10^{6} \\
(\mathrm{~cm} / \mathrm{sec})\end{array}$ & $\begin{array}{c}\mathrm{P}_{\mathrm{Cl}} \times 10^{7} \\
\left(\mathrm{~cm} / \mathrm{sec}^{2}\right)\end{array}$ \\
\hline 1 & 54.6 & 165 & 478 & 56.9 & 1.30 & - & 2.42 \\
2 & 69.6 & 101 & 391 & 28.2 & 1.06 & 1.81 & 0.59 \\
3 & 35.6 & 172 & 345 & 85.8 & 0.936 & 1.16 & 0.1 \\
4 & 11.5 & 176 & 220 & 141 & 0.596 & 0.306 & 0.1 \\
\hline
\end{tabular}

this case. the time constants were smaller than those for the anodal currents.

When only the $\mathrm{KCl}$ solution was used the exterior electrolyte and the concentration of electrolyte of the one side membrane was different from the other. the system gave essentially the same results as those mentioned above except for the smaller changes of current caused by the negative displacement of membrane potentials. Since the constant voltages measured with silver-silver chloride probes were affected by the voltage depression due to the electrical resistance of the solutions between the probes. the net membrane potentials by measuring the solution resistances as a function of the distance between the probes. ${ }^{67.8}$ The effect of the concentration polarization near the membrane surfaces would be ignored since a sudden removal of the applied potential caused a negligible polarization potential as compared with the resting potential. In addition. the time scale adopted for recording the current was so large that the capacity current was not insolved in measurement. For the initial resting state of various systems. the membrane conductance. the potentials and membrane permeability of each component are given in Table 1. The equation for calculation permeabilities were derived by using the formulations given by Kimizuka as follows." For the concentration cell of $\mathrm{KCl}$ solution:

$$
\begin{gathered}
P_{K^{-}}=\frac{1}{C_{i j}^{2}-C_{j}^{2}}\left(\frac{R T}{F^{2}} G_{+} C_{1}-\frac{R T}{F^{2}} G_{-} C_{l}\right) \\
P_{C l^{-}}=\frac{1}{C_{\hat{i}}^{2}-C_{j}^{2}}\left(\frac{R T}{F^{2}} G_{-} C_{1}-\frac{R T}{F^{2}} G_{-} C_{I}\right) \\
G=\left(G_{+} G_{-}\right)^{1 / 2} \\
\frac{F V_{0}}{R T}=\ln \frac{G_{-}}{G_{-}}
\end{gathered}
$$

Where $P_{K}$ and $P_{c l}$ denote the membrane permeabilities to potassium ion and chloride ion. respectively: $C_{0}$ and $C_{l^{*}}$ concentrations of $\mathrm{KCl}$ solution on the both side of membrane: $G_{ \pm}$is membrane conductance: $V_{i j}$. membrane potential at the resting state: $F$. Faraday constant: $R$. is gas constant: $T$. absolute temperature

Similarly the membrane permeabilities of components for the bi-ionic system composed of $\mathrm{KCl}$ and $\mathrm{CaCl}_{2}$ were written in the form:

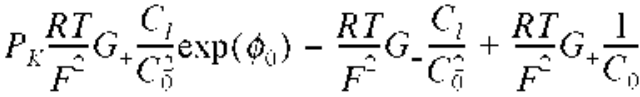

$$
\begin{aligned}
& P_{C l}=\frac{R T}{F^{2}} G_{-} \frac{1}{C_{l}} \exp \left(\frac{3}{2} \cdot \phi_{0}\right) \\
& \phi_{0}=\frac{R T}{F^{2}} G_{-} \frac{1}{C_{0}}-\frac{R T}{F^{2}} G_{-} \frac{1}{C_{0}} \exp \left(\phi_{0}\right) \\
& \phi_{i j}=F V_{i j} / R T
\end{aligned}
$$

and $G_{+} G_{-}$are calculated according to equations (3) and (4). If the following condition was maintained

$$
\begin{gathered}
q^{-} \gg p^{3} \\
3 P=\frac{P_{C l} C_{Q}}{P_{K} C_{Q}+P_{C l} C_{l}} . \quad 2 q=\frac{P_{C l} C_{l}}{P_{K} C_{0}+P_{C l} C_{l}}
\end{gathered}
$$

and $P_{C_{a}}$ indicates the membrane permeability to calcium: $C_{\alpha}$ and $C_{l}$, the concentrations of $\mathrm{KCl}$ and $\mathrm{CaCl}_{2}$ in nonmal, respectively. The Table $\mathrm{I}$ indicates that the membrane permeability to chloride ion was negligible as compared with those of sodium and calcium ions and hence the membrane used in this study is of the higher permselectivity for cations

In the subsequent treatment of non steady current, it is assumed that the membrane is uniform over its thickness $l$, and the ion fluxes are dependent upon position and time. The ion flux. $j \alpha$, of component $\alpha$ ion for isothermal system is given by the Nernst-Planck equation;

$$
j_{\alpha}=-D_{\alpha}\left(\frac{d C_{\alpha}}{d x}+\frac{z_{\alpha} F C_{\alpha}}{R T} \frac{d \psi}{d x}\right)
$$

Where $C_{\alpha}$ is the concentration of $\alpha$ ion within the membrane: $z_{\alpha}$ its charge: $D_{\alpha_{1}}$ its diffusion coefficient: $\psi$. the membrane potential: and $x$ denotes coordinate normal to the membrane. For the sake of simplicity the diffusion coefficient. $D_{\alpha}$. was assumed to be independent of the concentrations although it. in general. considered to be a complicated function of the concentration of all components. This was usually assumed by many investigators for describing the steady state of the membrane and electrolyte solution systems. jo

According to Goldmam. ${ }^{1]}$ the further simplification of the problem might be possible in the case where the assumption concerning with either the potential distribution or the 
concentration distribution of ion within membrane were proposed. The former assumes the microscopic electroneutrality at any position within the membrane, and the latter assumes that the potential gradient in the membrane is a constant.

The former case was studied exclusively by Theorell ${ }^{12}$ and the latter was preferred by Goldman and Hodgkin. ${ }^{13}$ However there is no theoretical reason for confirming which situation describes the characteristics of system more exactly: In view of the fact that the current voltage relations obtained with the non-steady currents at constant times can be reproduced by the expression based on the assumption of constant electrical field. it seems reasonable to apply this assumption for the non stationary sy'stems. ${ }^{14}$

Denoting $x$ ant $t$ for the coordinate normal to the membrane and time. respectively. the equation of continuity is given by

$$
\frac{\partial I_{\alpha}}{\partial x}=-\frac{\partial C_{\alpha}}{\partial t}
$$

which represents the conservation of mass for the component $\alpha$. With the assumption of constant electrical field and by equation (12). the equation of flux expressed by NernstPlanck equation is reduced to

$$
\frac{\partial j_{\alpha}}{\partial T}=\frac{\partial^{2} C_{\alpha}}{\partial x^{2}}+2 \phi_{\alpha} \frac{\partial C_{\alpha}}{\partial x}
$$

where $\phi_{\alpha}$ and $T$ are given by following equations:

$$
\begin{gathered}
2 \phi_{\alpha}=\frac{z_{\alpha} F}{R T} \frac{d \psi}{d x} \\
T=\int_{0}^{t} D_{\alpha} d t^{\prime}
\end{gathered}
$$

in which $\phi_{\alpha}$ is independent of position owing to the present assumption.

The generalized diffusion equation represented by equation (13) could be solved with the initial condition:

$$
\begin{aligned}
& C_{\alpha}(x, 0)=-\frac{j_{\alpha \mid}}{2 \phi_{\alpha \mid j} D_{\alpha}}+\left(\frac{j_{\alpha \mid 1}}{2 \phi_{\alpha \mid j} D_{\alpha}}+C_{\alpha(0)}\right) \exp \left(-2 \phi_{\alpha \mid !} x\right) \\
& 0 \leq x \leq 1
\end{aligned}
$$

And with the boundary condition:

$$
\begin{aligned}
& C_{\alpha}(0 . T)=C_{\alpha i} \\
& C_{\alpha}(I . T)=-\frac{j_{\alpha \beta s}}{2 \phi_{\alpha} D_{\alpha}}+\left(\frac{j_{\alpha \alpha s}}{2 \phi_{\alpha \alpha} D_{\alpha}}+C_{\alpha(1)}\right) \exp \left(-2 \phi_{\alpha l}\right) \\
& 0 \leq T \leq \infty
\end{aligned}
$$

where $C_{\alpha i j}$, the concentration of ion $\alpha$ at $x=0$. and $T=0, j \alpha$ and $j_{\alpha}$, the fluxes at $T=0$ and $T=\infty$, respectively and $\phi$ corresponds to $\phi_{\alpha}$ given by equation (14) at the initial resting state. The solution for equation (13) was obtained in the polynomial form as follows:

$$
\begin{aligned}
& C_{\alpha}(x, T)=\frac{j_{\alpha x s}}{2 \phi_{\alpha} D_{\alpha}}+\left(\frac{j_{\alpha s}}{2 \phi_{\alpha} D_{\alpha}}+C_{\alpha, 1)}\right) e^{-2 \phi_{\alpha}} \\
& +\frac{2 \pi e^{-\phi_{\alpha z}}}{1^{2}} \sum_{n=1}^{\infty} \frac{n \sin \left(\frac{n \pi x}{1}\right)}{\zeta_{\alpha n} \eta_{\alpha n}} \\
& {\left[\left(C_{\alpha i 1}+\frac{j_{\alpha i}}{2 \phi_{\alpha(1)} D_{\alpha}}\right) \times\left(\eta_{\alpha i n}-\zeta_{\alpha n}\right)\right.} \\
& -\left\{\eta_{\alpha n}\left(\frac{j_{\alpha i 1}}{2 \phi_{\alpha \alpha} D_{\alpha}}-\frac{j_{\alpha s}}{2 \phi_{\alpha} D_{\alpha}}\right) e^{\left.\phi_{\alpha}\right\}}\right. \\
& +\eta_{\alpha n}\left(C_{\alpha i}+\frac{j_{\alpha \omega}}{2 \phi_{\alpha} D}\right) e^{-\phi_{\alpha, l}}-\zeta_{\alpha n}\left(C_{\alpha 0}+\frac{j_{\alpha i}}{2 \phi_{\alpha i 0} D_{\alpha}}\right) \\
& \left.\left.\times e^{\left.i \phi_{(\prime)}-2 \phi_{(r)}\right) l}\right\}(-1)^{n}\right] e^{\xi_{c o t} T}
\end{aligned}
$$

in which the following abbreviations were used:

$$
\begin{aligned}
& \zeta_{\alpha n}=-\left\{\phi_{\alpha}^{2}+\left(\frac{n \pi}{1}\right)^{2}\right\} \\
& \eta_{\alpha n}=\zeta_{\alpha n}+4\left(\phi_{\alpha} \phi_{\alpha i 1}-\phi_{\alpha \dot{ }}^{2}\right)
\end{aligned}
$$

and $\eta$ denotes positive integers. For large $T$. the equation (19) may be expressed by the term with $n=1$. Hence, we obtain

$$
\begin{aligned}
& C_{\alpha}(x, T)=-\frac{j_{\alpha s x}}{2 \phi_{\alpha} D_{\alpha}}+\left(\frac{j_{\alpha s}}{2 \phi_{\alpha} D_{\alpha}}+C_{\alpha 10}\right) e^{-2 \phi_{\alpha}} \\
& +\frac{2 \pi}{1^{2}} e^{-\zeta_{2, d} T-\phi_{c} x} \sin \left(\frac{\pi x}{1}\right) \\
& {\left[\frac { 1 } { \zeta _ { \alpha l } } \left\{C_{\alpha(1)}+\frac{j_{\alpha 1)}}{2 \phi_{\alpha(1)} D_{\alpha}}+\left(\frac{j_{\alpha(1)}}{2 \phi_{\alpha(1)} D_{\alpha}}-\frac{j_{\alpha s}}{2 \phi_{\alpha} D_{\alpha}}\right) e^{\phi_{\alpha(l)}}\right.\right.} \\
& \left.+\left(C_{\alpha i j}+\frac{j_{\alpha \alpha}}{2 \phi_{\alpha} D}\right) e^{-\phi_{\alpha t}}\right\}-\frac{1}{\eta_{\alpha l}}\left(C_{\alpha i j}+\frac{j_{\alpha(j)}}{2 \phi_{\alpha(!} D_{\alpha}}\right) \\
& \left.\times\left\{1+e^{\left.\left(\varphi_{x}-2 \psi_{x}\right)\right\}}\right\}\right]
\end{aligned}
$$

where $\zeta_{\alpha I}$ and $\eta_{\alpha 1}$ are equal to those of equations (20) and (21) at $n=1$. respectively. By the substituting the equation (22) to equation (11) it is possible to represent the flux equation as the explicit function of time and position:

$$
\begin{aligned}
& j_{\alpha}=j_{\alpha=x}-\frac{2 \pi D}{1^{2}} e^{-\xi_{\alpha x} T-y_{x} x}\left\{\phi_{\alpha} \sin \left(\frac{\pi x}{1}\right)+\frac{\pi}{1} \cos \left(\frac{\pi x}{1}\right)\right\}
\end{aligned}
$$

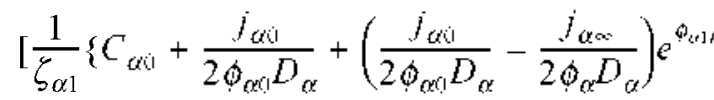

$$
\begin{aligned}
& \left.+\left(C_{\alpha \mid)}+\frac{j_{\alpha \infty}}{2 \phi_{\alpha} D_{\alpha}}\right) e^{-\phi_{\alpha 1}}\right\}-\frac{1}{\eta_{\alpha 1}}\left(C_{\alpha(1}+\frac{j_{\alpha(1}}{2 \phi_{\alpha \mid 1} D_{\alpha}}\right) \\
& \left.\times\left\{1+e^{\left.\left[\phi_{\alpha}-2 \phi_{\alpha,}\right)\right\}}\right\}\right]
\end{aligned}
$$

Equation (23) indicates that the non steady flow of ion $\alpha$ can be described completely provided the initial and final steady states. the diffusion coefficient of the ion and applied potential are given. The summation of the fluxes for the all constituent ions the total currents: 


$$
I=\sum_{\alpha}^{r} z_{\alpha \alpha} F j_{\alpha}, I_{0}=\sum_{\alpha}^{r} z_{\alpha} F j_{\alpha 10}, \quad I_{\infty}=\sum_{\alpha}^{r} z_{\alpha} F_{j} j_{\alpha \infty}
$$

where $I, I_{0}$ and $I_{s}$ are the total currents at the finite, zero and infinite times. respectively. Combining the equation (24) with the equation (23) and eliminating $I \propto$ we have the time dependence of the non steady current at the membrane boundary: $x=0$. as follows;

$$
\begin{aligned}
& I-I_{0}=\frac{\pi^{2} F}{1} \sum_{\alpha}^{r} z_{\alpha} \\
& \times\left[\frac{\left(\frac{j_{\alpha 0}}{\phi_{\alpha 0}}-\frac{j_{\alpha 10}}{\phi_{\alpha}}\right)\left(\mathrm{l}+e^{\phi_{\alpha 1}}\right)+\left(2 D_{\alpha} C_{\alpha(0}+\frac{j_{\alpha \infty}}{\phi_{\alpha}}\right)\left(1+e^{-\phi_{\alpha l}}\right)}{\phi_{\alpha}^{2} \mathrm{~L}^{2}+\pi^{2}}\right. \\
& \left.-\frac{\left(2 D_{\alpha} C_{\alpha(0}+\frac{j_{\alpha 0}}{\phi_{\alpha 0}}\right)\left\{1+e^{\left.\left(\phi_{\alpha}-2 \phi_{\alpha 0}\right\}\right]}\right\}}{\left(\phi_{\alpha}-2 \phi_{\alpha 1}\right)^{2} 1^{2}+\pi^{2}}\right] \\
& \times\left[e^{-\left\{\phi_{\omega}^{2}-\left(\frac{\pi}{i}\right) ; D_{\omega} t\right.}-1\right]
\end{aligned}
$$

after substituting the equations (15). (20) and (21) for T. $\zeta$ and $\eta$. respectively.

The resting potential, $V_{0}$ and the applied potential. $V$ are introduced into equation (25) through the following relations:

$$
\phi_{\alpha !}=\frac{z_{\alpha} F}{2 R T} \frac{V_{0}}{l} . \quad \phi_{\alpha}=\frac{z_{\alpha \alpha} F}{2 R T} \frac{V}{l} .
$$

In equation (25) the relaxation of current is expressed as the deviation from the initial current and the difference between the initial and funal steady currents are determined by the extrapolation to the infinite time. It was obvious that the time constant for the relaxation of current was parallel to the applied potential and proportional to the diffusion coefficient as well as the reciprocal of membrane thickness. On the other hand the amount of variation in the current was found to be depend on the many factors, such as applied potential, diffusion coefficient. initial concentration of components within membrane. the resting potential and the initial and final steady fluxes. in more complicated manner. ${ }^{15}$

In order to estintate the flux at resting state, the following equation could be available: ${ }^{16}$

$$
j_{\alpha 0}=-\frac{P_{\alpha}}{G^{\mid F_{d}}}\left(C_{\alpha}^{(i)} G_{-}^{F_{d}}-C_{\alpha}^{J} G_{-}^{\mid E_{d}}\right)
$$

where $C^{\prime}{ }_{x}$ and $C^{l}$ are bulk concentrations of ion $\alpha$ on the both sides of membrane and $P_{a x}$ is the membrane permeability to the ion $\alpha$. Assuning that in the membrane. the potential gradient is a constant and the diffusion coefficient, $D_{1 x}$ is a constant independent of concentration $P_{x x}$ may be reduced to

$$
P_{\alpha}=\frac{z_{\alpha} F V / 2 R T}{\sinh h z_{\alpha} F V / 2 R T} \times \frac{D_{\alpha}}{l} e^{-\pi_{\alpha} R T}
$$

which. provided $z_{\alpha} F V / 2 R T$. is small as compared with unity: is reduced to

$$
P_{\alpha} \triangleq \frac{D}{l} e^{-\eta_{\alpha} R T} \cong \frac{\bar{D}_{\alpha}}{l}
$$

where $\eta_{\alpha}$ indicates excess free energy of the ion $\alpha$. Inserting equation (29) into equation (27). we obtain the next equation (30)

$$
j_{\alpha i}=-\frac{\bar{D}_{\alpha}}{G^{|-\alpha|}}\left(C_{\alpha}^{+0} G_{-}^{\mid F_{d}}-C_{\alpha}^{l} G_{+}^{\mid=d}\right)
$$

Thus the fluxes at rest and the diffusion coefficients could be estimated from the data shown in Table 1 . The time courses

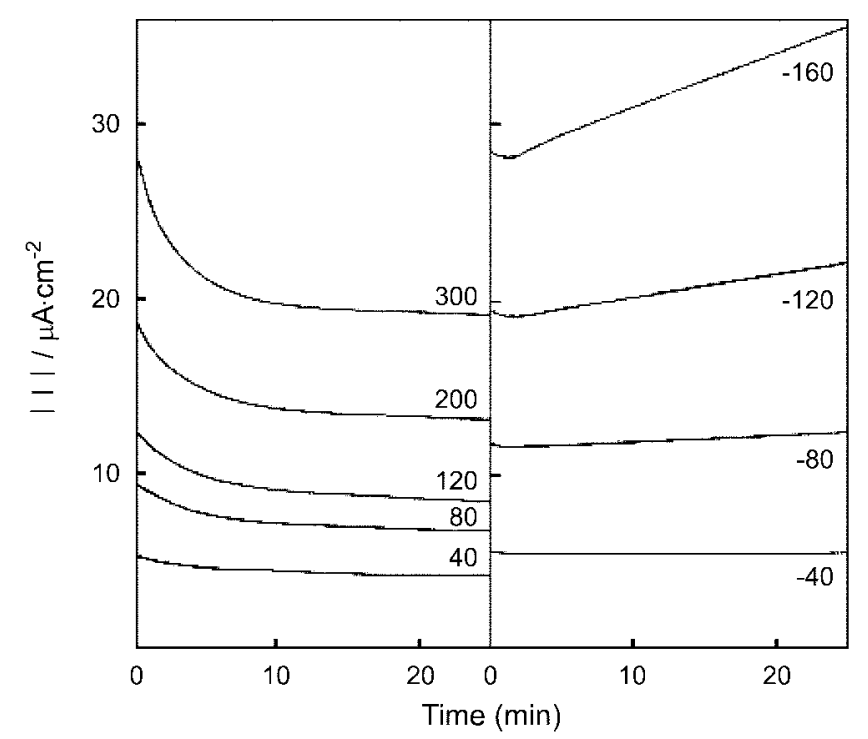

Figure 2. Records of non steady current under a constant applied voltage for system no. 3. The displacement of membrane potential is given in millivolts by the number attached to each records.

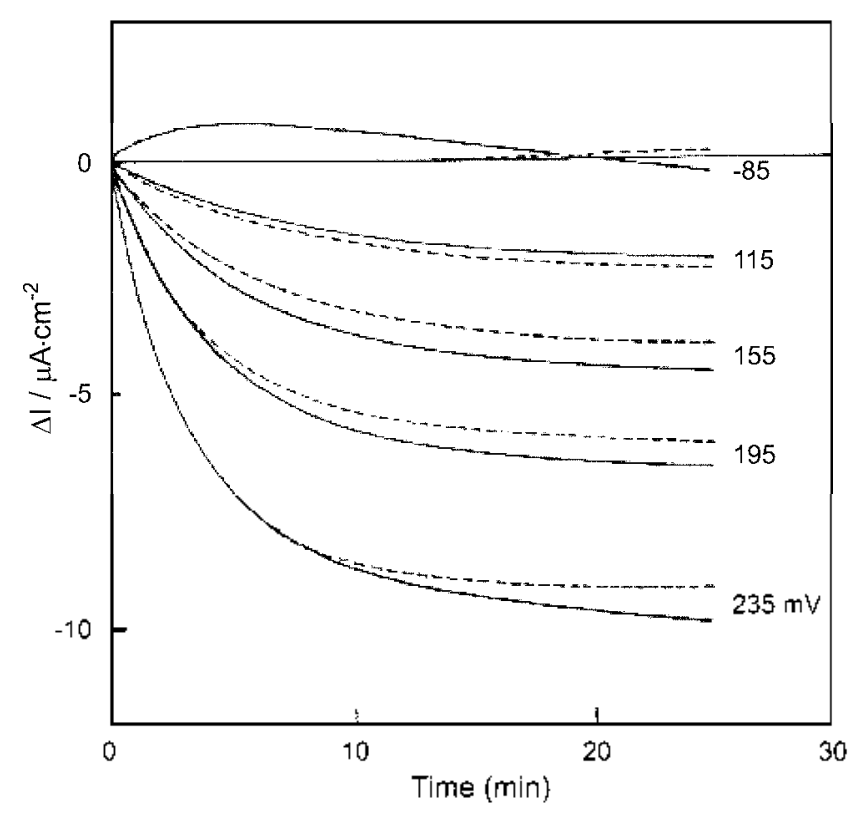

Figure 3. The records present an applied potential; solid line, experimental walue and dashed line, calculated used. 


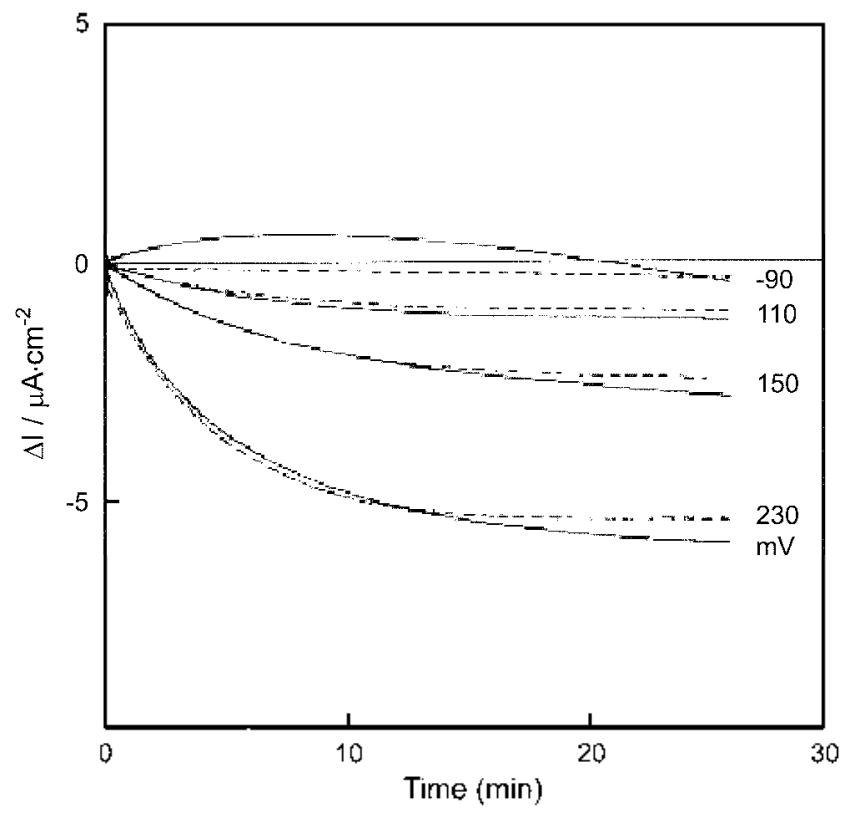

Figure 4 . Time course of non steady curent for the electrolyte solution system no.2. Solid lines are experinental value and dashed lines are calculated value according to equation (23).

of the non-steady currents measured at various applied voltages are illustrated in Figures 2. 3. 4 and 5 for the different systems. respectively. The calculated diffusion coefficients from these results were smaller than those estimated by equation (29) for the permeabilities in Table 1. This fact seems to be due to the different treatment from the previous one which was determined without the constant field assumption. However. the permeability ratio for a given system should be preferred that determined with the method of Kimizuka ${ }^{17.18}$ rather than that according to Hodgkin and Katz.

Therefore it was possible to calculate the diffusion coefficients with the time constants and equation (25). provided the ratio of diffusion coefficients is according to equation (29). These diffusion coefficients and fluxes of potassium and calcium at rest were shown in Table 2 for the various systems. The corresponding quantities of chloride ion were so small that they could not calculate exactly.

As $C_{\infty}$ is the concentration of ion $\alpha$ at membrane boundary in the initial steady state. it is unlikely reasonable to estimate $C_{a}$ by assuming Donnan equilibrium. In the Figures 3,4 and 5 , it was found that the final current. $J_{\alpha \infty}$

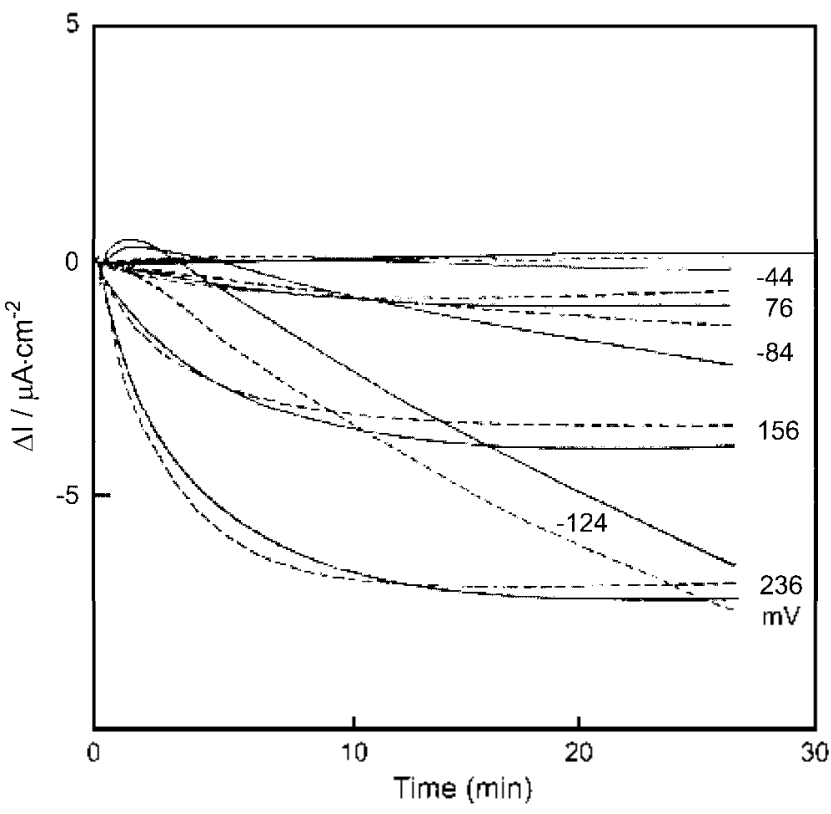

Figure 5. Time course of non steady current for system no.3 and the numbers attached to the records represent an applied potential; solid lines are experimental data, dashed lines are calculated value.

could not be estimated for case of the negative applied potentials. Consequently: the values of $C_{x x i}$ and $J_{(x)}$ are necessary to determine so that they could reproduce the experimental data. The values of $C_{x \rightarrow i}$ and $J_{t x<}$ estimated in such way are given in Table 3 to 6 . Thus the time courses of the relaxation current for the various applied potential calculated according to equation (25) could reproduce the experimental results as shown in Figures 2 to 5 . These

Table 3. The ion flus and membrane potential of potassium ion for the $01 \mathrm{~N}-\mathrm{KCl}$ rs. $0.001 \mathrm{~N}-\mathrm{KCl}$ solution system through composite charged membrane

\begin{tabular}{cc}
\hline $\mathrm{V}(\mathrm{mV})$ & $j_{K} \times 10^{12}\left(\mathrm{~mole} / \mathrm{cm}^{2} \mathrm{sec}^{-1}\right)$ \\
\hline 195 & -12.3 \\
155 & -8.85 \\
115 & -6.44 \\
95 & -4.68 \\
65 & -1.98 \\
35 & 1.28 \\
-5 & 6.62 \\
-85 & 20.4 \\
\hline
\end{tabular}

Table 2. The diffusion coefficient, ionic concentration and ionic flux value at resting state for potassium ion and calcium ion through composite charged membrane and system no. 1:0.01 N-KClo.001 N-KC1, 2:0.01 N-KCl/0.001 N-CaCl, 3:0.01 N-KCV/0.01 N-CaCl, 4: $0.01 \mathrm{~N}-\mathrm{KCl} / 0.1 \mathrm{~N}-\mathrm{CaCl}_{2}$

\begin{tabular}{|c|c|c|c|c|c|c|}
\hline $\begin{array}{c}\text { System } \\
\text { no. }\end{array}$ & $\begin{array}{l}\mathrm{D}_{\mathrm{K}} \times 10^{4} \\
\left(\mathrm{~cm}^{\circ} \mathrm{sec}\right)\end{array}$ & $\begin{array}{l}\mathrm{D}_{\mathrm{la}} \times 10^{10} \\
\left(\mathrm{~cm}^{2} \mathrm{sec}\right)\end{array}$ & $\begin{array}{l}\mathrm{C}_{\mathrm{K}} \times 10^{2} \\
(\text { mole } / 1)\end{array}$ & $\begin{array}{l}\mathrm{C}_{\mathrm{Ca}} \times 10^{3} \\
(\text { mole } / 1)\end{array}$ & $\begin{array}{c}j_{\mathrm{k}} \times 10^{1 / 2} \\
\left(\mathrm{~mole} / \mathrm{cm}^{2} \mathrm{sec}^{-1}\right)\end{array}$ & $\begin{array}{c}j_{\mathrm{Ca}} \times 10^{15} \\
\left(\mathrm{~mole} / \mathrm{cm}^{2} \mathrm{sec}^{-1}\right)\end{array}$ \\
\hline 1 & 1.43 & - & 4.8 & - & 13.6 & - \\
\hline 2 & 0.93 & 1.58 & 4.4 & 0.15 & 11.9 & -2.06 \\
\hline 3 & 1.37 & 1.70 & 2.1 & 1.2 & 9.17 & -75.2 \\
\hline 4 & 1.85 & 0.95 & 1.7 & 11.0 & 3.95 & -101.0 \\
\hline
\end{tabular}


Table 4. The fluxes of potassium ion and calcium ion at and membrane potential for $0.01 \mathrm{~N}-\mathrm{KCl}$ v. $0.001 \mathrm{~N}-\mathrm{CaCl}_{2}$ solution system through composite charged membrane

\begin{tabular}{ccc}
\hline $\mathrm{V}(\mathrm{mV})$ & $j \mathrm{~K} \times 10^{12}\left(\mathrm{~mole} / \mathrm{cm}^{\left.\hat{*} \mathrm{sec}^{\mathrm{l}}\right)}\right.$ & $j \mathrm{Ca} \times 10^{14}\left(\mathrm{~mole} / \mathrm{cm}^{\left.\hat{*} \mathrm{sec}^{1}\right)}\right.$ \\
\hline 230 & -5.43 & -39.1 \\
150 & -2.72 & -24.2 \\
110 & -0.94 & -18.9 \\
30 & 5.28 & -6.74 \\
-10 & 10.9 & -1.56 \\
-50 & 18.0 & 0.08 \\
-90 & 26.7 & 2.73 \\
\hline
\end{tabular}

Table 5. The fluxes of potassium and calcium ion at $t=\infty$ against membrane potential value for $0.01 \mathrm{~N}-\mathrm{KCl} v 5.0 .01 \mathrm{~N}-\mathrm{CaCl}_{2}$ solution system through composite charged membrane

\begin{tabular}{rcc}
\hline $\mathrm{V}(\mathrm{mV})$ & $j \times 10^{12}\left(\mathrm{~mole} / \mathrm{cm}^{2} \mathrm{sec}^{-1}\right)$ & $j \mathrm{ca} \times 10^{12}\left(\mathrm{~mole} / \mathrm{cm}^{2} \mathrm{sec}^{-1}\right)$ \\
\hline 236 & -16.5 & -45.2 \\
156 & -9.94 & -37.7 \\
76 & -2.61 & -13.8 \\
-4 & 2.15 & -0.98 \\
-44 & 8.20 & 1.39 \\
-84 & 15.4 & 2.42 \\
-124 & 23.1 & 3.36 \\
\hline
\end{tabular}

Table 6. The fluxes of potassium ion and calcium ion against membrane potential for $0.01 \mathrm{~N}-\mathrm{KCl}$ vs. $0.1 \mathrm{~N}^{-\mathrm{CaCl}_{2}}$ solution system through charged membrane

\begin{tabular}{rcc}
\hline $\mathrm{V}(\mathrm{mV})$ & $j \mathrm{~K} \times 10^{12}\left(\mathrm{~mole} / \mathrm{cm}^{2} \mathrm{sec}^{-1}\right)$ & $j \mathrm{ca} \times 10^{12}\left(\mathrm{~mole} / \mathrm{cm}^{2} \mathrm{sec}^{-1}\right)$ \\
\hline 212 & -29.0 & -23.0 \\
132 & -20.0 & -14.3 \\
72 & -12.4 & -7.78 \\
-28 & 2.98 & -2.36 \\
-68 & 11.5 & 0.99 \\
-108 & 20.6 & 1.46 \\
-148 & 30.0 & 1.96 \\
\hline
\end{tabular}

results indicate that the equation (25) describes the essential feature of the relaxation phenomena.

The $C_{1}, \alpha$ were found to be proportional to the bulk concentrations of calcium ion. Through the bulk concentration of the one side of the membrane was constant. the ion concentration within the membrane seemed to depend on the calcium ion concentration present in the order side. This can be deduced in terms of the variation of $C_{\text {ijK }}$ shown in Table 2 .

\section{Conclusions}

It is noted in membrane equation that the $C_{\alpha}$ value contribute to the amount of the relaxation current considerably and it may be concluded that the relaxation of current were controlled by the ion transports from the interior of the membrane to the external solution. For the $\mathrm{KCl}-\mathrm{CaCl}_{2}$

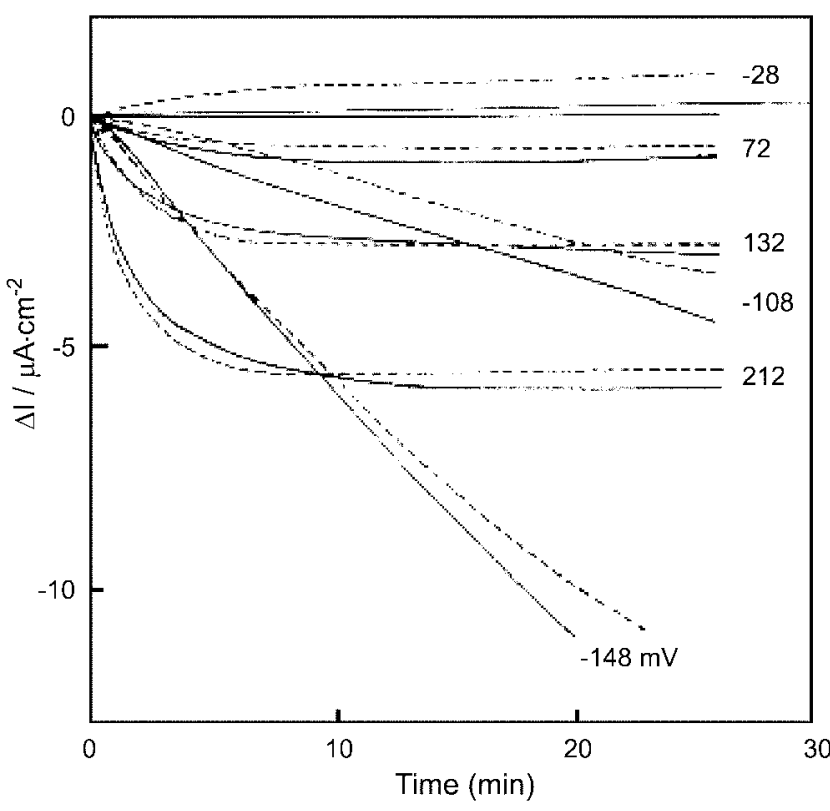

Figure 6. The time collse of non steady current for system no.4 and the numbers attached to the records represent an applied potential and solids are experimental data and dashed are calculated value.

şistems, the time course of current was mainly governed by potassium ion transport when the $\mathrm{CaCl}_{2}$ solution side was positively polarized and vice versa.

Acknowledgement. This work was supported by the Dongguk University Research Fund.

\section{References}

1. Hodgkin. A. L.: Huxley, A. F. J. Phnsiol 1952, 117, 500.

2. Baker, P. E.: Hodghin, A. L.: Shaw. T. I. J. Physiol 1962. 164. 330

3. Chandler. W. K.: Meves. H. JPhusiol 1965. 180.788 \& 821.

4. Kirkwood. J. G. Ion Transpont derass Mentbrane: Clark. H. T.. Ed. Academic press: 1954 ; 119

5. Neihof. R. J Phys. Chent 1954. 58,916

6. Lakshiminaravanaiah. N. J. Polymer Sci. 1960. 46. 529.

7. Lakshiminarayanaiah. N. Subcell, Biochem. 1979.6.401.

8. Lakshiminarayanaiah. N. Transport Phenomena in Membrone: Academic press: N.Y. 1969: Chap. 5.

9. Kimizuka. H. J. Theoret. Biol. 1966. 13, 145

10. Nagata, Y:, Kohara, K: Yang. W: Yamauchi, A.: Kimizuka, H Bull. Chem. Soc. Jpn. 1988, 61,793.

11. Goldmant. D. E. J. Gen. Plnsiol. 1944. 27.37

12. Teorell. T. Progr: Bioplys. 1953. 3. 305.

13. Hodgki11. A. L.: Katz. B. J. Plnsiol. 1949. 108.37.

14. Kedem, O: Katchalsky: A. Trans. Faradan Soc. 1963. 59 1918 .

15. Schultz, S. G. Basic Principle of Membrane Transport, Cambridge Univ, press: 1980: Chap 4. 6.

16. Siddiqi. F. A.: Alvi. N. I. J. Hembrame Sci. 1989. 46. 185.

17. Yang. W: Yamauchi. A.: Kimizuka. H. J. Mentrane Sci. 1992. 70.277

18. Hirata, Y; Deguchi. S.: Yamauchi, A.; Kimizuka, H. Nippon Kagaku Kaishi 1988. 1536. 